Supporting Information for

\title{
Finely Tuning Free Volume Architecture in Iptycene-containing Polyimides for Highly Selective and Fast Hydrogen Transport
}

Shuangjiang Luo, ${ }^{\dagger}$ Jennifer R. Wiegand, ${ }^{\dagger}$ Barbara Kazanowska, ${ }^{\dagger}$ Cara M. Doherty, ${ }^{\ddagger}$ Kristina Konstas, ${ }^{\ddagger}$ Anita J. Hill, ${ }^{\ddagger}$ Ruilan Guo ${ }^{\dagger *}$

${ }^{\dagger}$ Department of Chemical and Biomolecular Engineering, University of Notre Dame, Notre Dame, IN 46556, United States

*The Commonwealth Scientific and Industrial Research Organization (CSIRO) Manufacturing, Private Bag 10, Clayton South, Victoria 3169, Australia

* Corresponding author: +1-574-631-3453 (tel), +1-574-631-8366 (fax), rguo@,nd.edu 

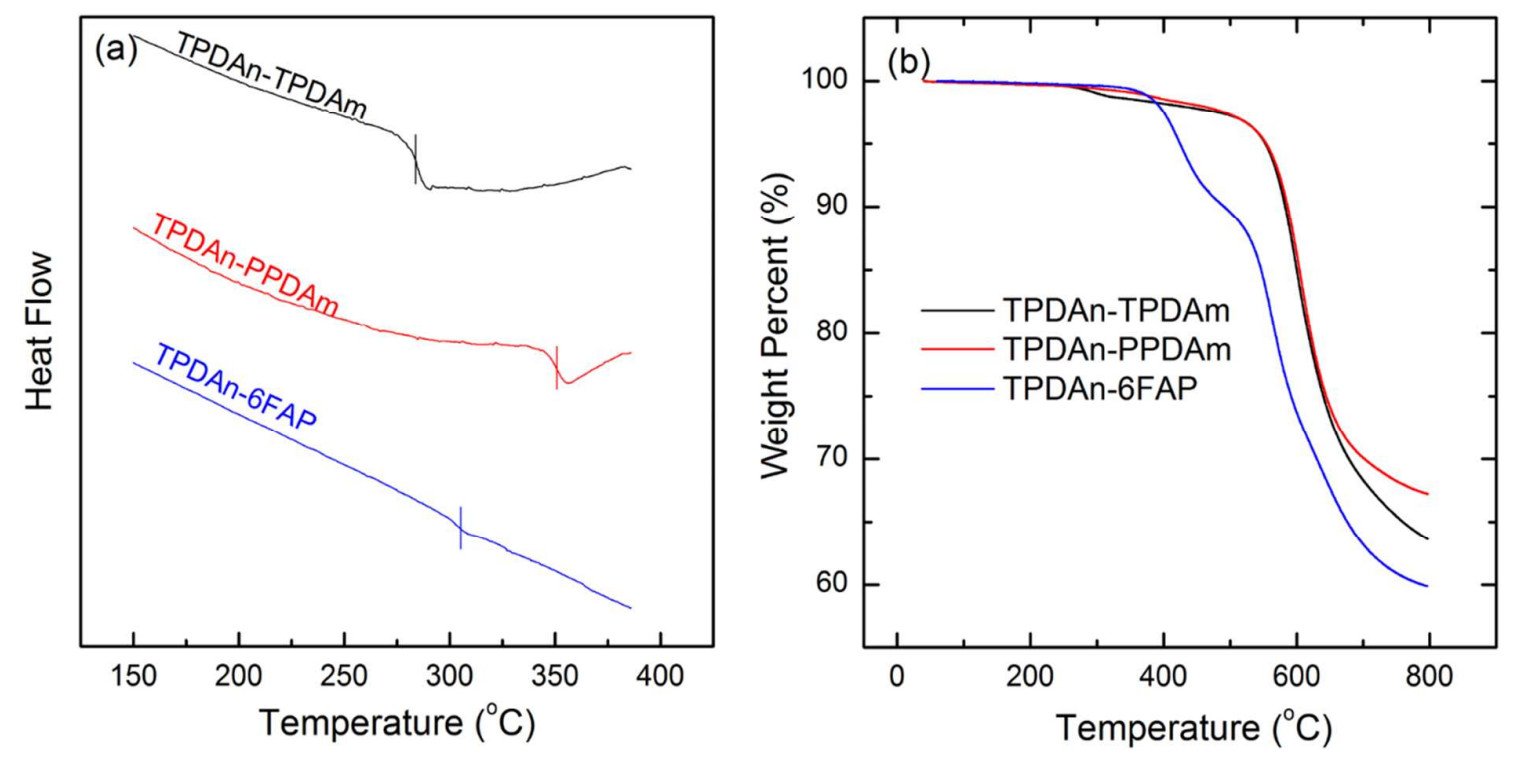

Figure S1. (a) DSC and (b) TGA curves of iptycene-based polyimides.
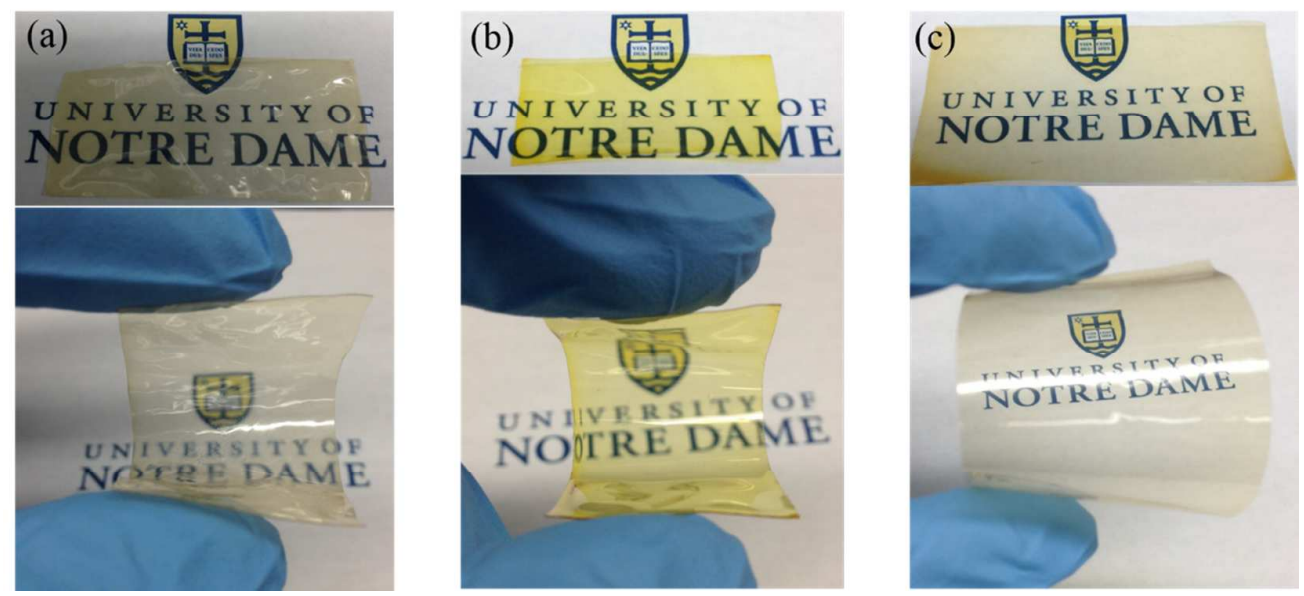

Figue S2. Photographs of the thin membranes of (a) TPDAn-TPDAm, (b) TPDAn-PPDAm and (c) TPDAn-6FAP polyimide. 

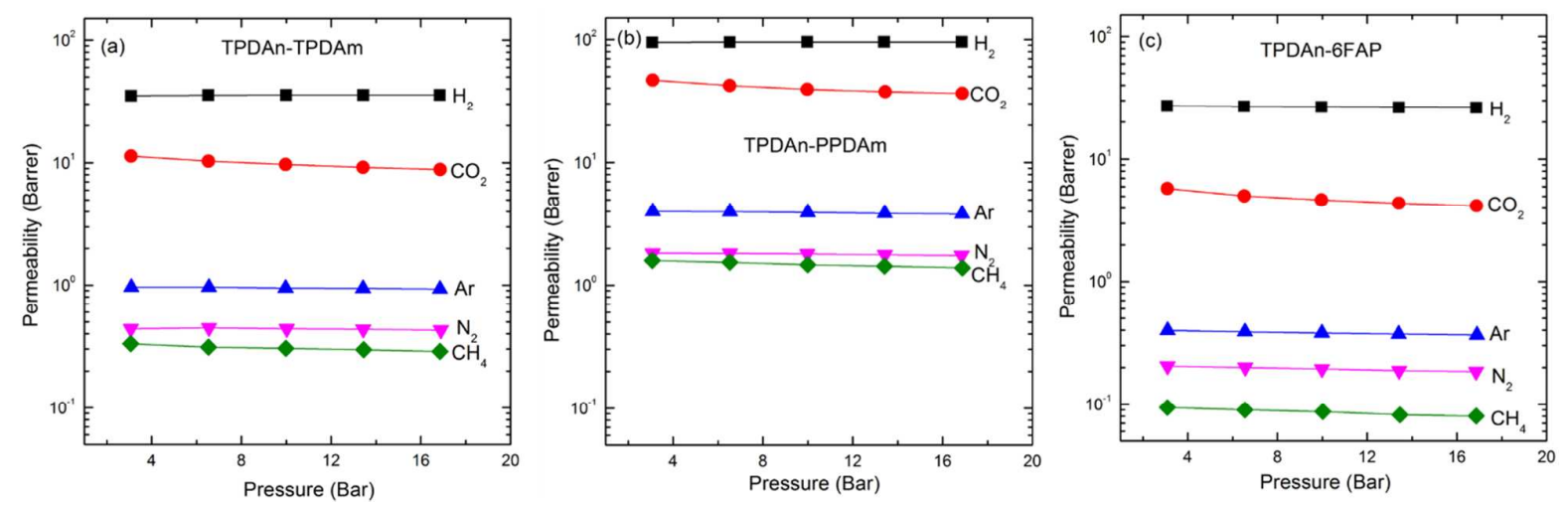

Figure S3. Pure gas permeabilities of the iptycene-based polyimides as a function of feed pressure.
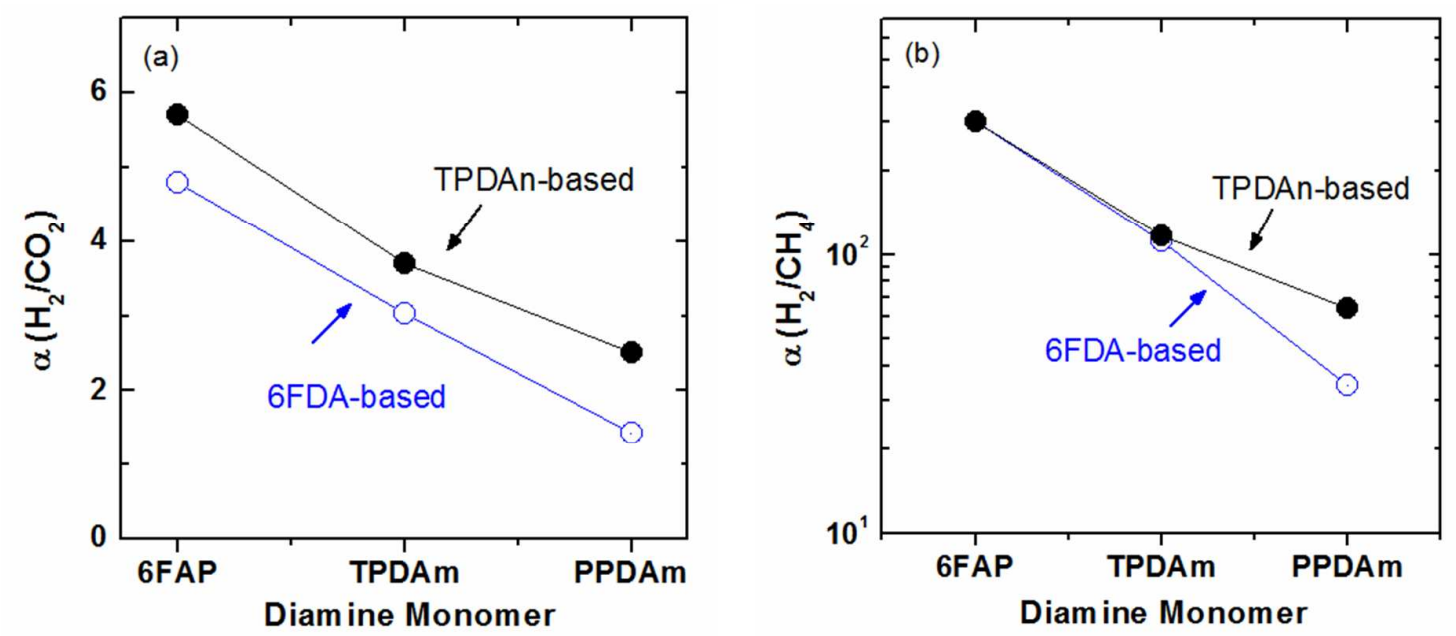

Figure S4. Effect of the diamine and dianhydride structure on (a) $\mathrm{H}_{2} / \mathrm{CO}_{2}$ and (b) $\mathrm{H}_{2} / \mathrm{CH}_{4}$ selectivities for iptycene-based polyimides. 